\title{
Natural history of early gastric cancer: series of 21 cases
}

\section{()ㅛ $\ominus$}

\begin{abstract}
Authors
Hiroyoshi Iwagami ${ }^{1}$, Ryu Ishihara ${ }^{1}$, Kentaro Nakagawa ${ }^{1}$, Masayasu Ohmori ${ }^{1}$, Kenshi Matsuno ${ }^{1}$, Shuntaro Inoue ${ }^{1}$,

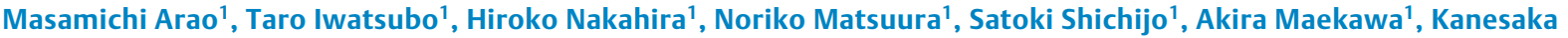

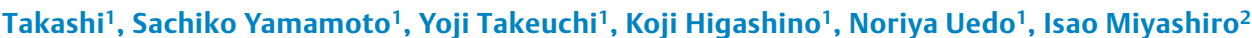

Institutions

1 Division of Gastrointestinal Oncology, Osaka International Cancer Institute, Osaka, Japan

2 Division of Cancer Control Center, Osaka International Cancer Institute, Osaka, Japan

submitted 26.2.2018

accepted after revision 22.8 .2018

Bibliography

DOI https://doi.org/10.1055/a-0746-3329 |

Endoscopy International Open 2019; 07: E43-E48

(c) Georg Thieme Verlag KG Stuttgart · New York ISSN 2364-3722

Corresponding author

Ryu Ishihara, MD, Division of Gastrointestinal Oncology, Osaka International Cancer Institute, 3-1-69 Otemae,

Chuo-ku, Osaka, 541-8567, Japan

Fax: +8166945 1902

ryu1486@gmail.com

\section{ABSTRACT}

Background and study aims While knowledge of the natural history of early gastric cancer (EGC) may be useful in relevant clinical situations, few relevant reports are available. Therefore, we investigated the progression of EGC. We gathered data regarding 114 cases of EGC from 2005 to 2015 from a hospital cancer registry and analyzed 21 lesions that fulfilled five inclusion criteria. Deep progression was defined as submucosal invasion by a mucosal tumor and proper muscle invasion by a submucosal tumor. Lateral progression was defined as $\geq 20 \%$ increase in size. During median follow-up of 23 months, one of 18 mucosal tumors showed deep progression and six showed lateral progression. Of three submucosal tumors, two showed deep progression and three showed lateral progression. Our study suggests that a certain proportion of mucosal cancers can lie dormant for several years. Further large-scale studies in a multicenter setting should overcome the limitations of this study.

\section{Introduction}

Early gastric cancer (EGC) carries a definite risk of progression and affects survival $[1,2]$. However, we sometimes experience a subset of gastric cancer (GC) which progresses very slowly. Information regarding the natural history of EGC may be useful in various clinical situations because older patients, those with limited life expectancy, or those at high operative risk should be informed about the expected outcomes for non-operative management of GC.

Non-operative management is divided into endoscopic treatment, no treatment with follow-up, and no follow-up. Even if curing the lesion is difficult, endoscopic treatment may be performed at the patient's request because tumor reduction may delay appearance of symptoms and relieve pain. No followup is chosen when EGC is considered not to be a prognostic factor for reasons such as other cancers or diseases. No treatment with follow-up is selected when endoscopic treatment is not performed for reasons such as the patient's advanced age or poor general condition. In this case, the natural history of EGC helps treatment decision-making. However, there have been few reports on the natural history of EGC, especially regarding early-stage cancer.

Therefore, we aimed to investigate progression of EGC in Japan, mainly mucosal cancer (i. e., mucosal high-grade neoplasia [HGN] in Western countries) and to clarify the natural history of EGC based on endoscopic follow-up data.

\section{Case reports}

This was a retrospective study conducted at the Osaka International Cancer Institute. Patient inclusion criteria were as follows: (1) endoscopically diagnosed mucosal or submucosal lesion; (2) histologically diagnosed adenocarcinoma on biopsy specimen; (3) no surgical or endoscopic resection for more than 1 year; (4) no exposure to chemotherapy or radiation for GC or other primary cancers; and (5) one or more endoscopic follow-ups.

Follow-up endoscopic examinations were conducted mainly to monitor progression of cancer. Depth of tumor invasion was 


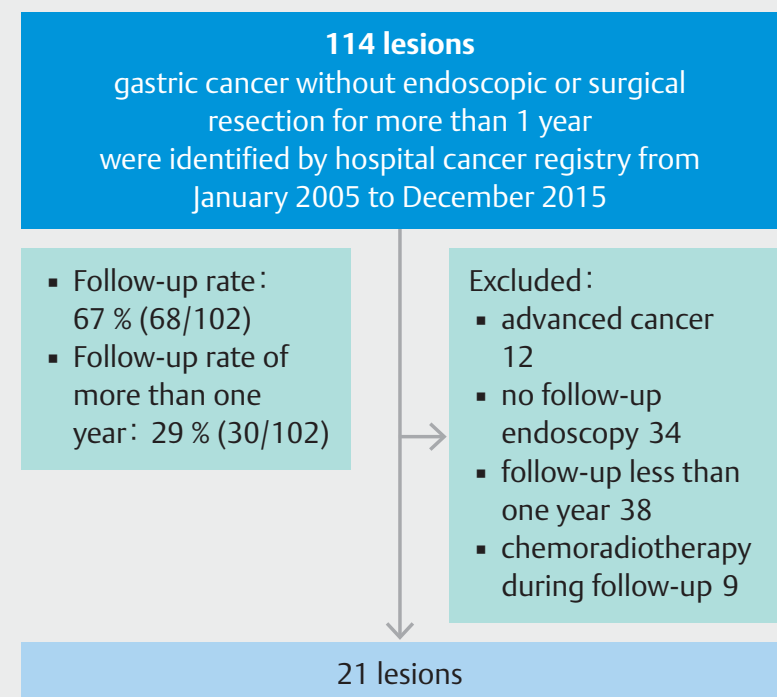

Fig. 1 Diagram showing patient enrollment.

determined by endoscopic findings, such as thickness of the lesion, stiff protrusion, marginal elevation, remarkable redness, and irregularity of the surface $[3,4]$. Deep progression (DP) was defined as submucosal invasion by a mucosal tumor and proper muscle invasion by a submucosal tumor. Lateral progression (LP) was defined as $\geq 20 \%$ increase in size. The size of lesions was estimated by comparison with the known diameter of an endoscope or open forceps.

Vital status and causes of death were confirmed by medical charts. Patients who died from causes other than GC were regarded as censored at the time of death. Patients with unknown vital status were also treated as censored at the time when they were last known to be alive. Time to progression was measured from date of diagnosis to first date of deep progression or lateral progression. The time-to-progression curve was generated using the Kaplan-Meier method. Statistical analysis was carried out by R version 3.3.3 (http://www.r-project.org).

Twenty-one lesions fulfilled all the inclusion criteria ( $\vee$ Fig. 1). A summary of characteristics of the patients and lesions is shown in $>$ Table 1 . The follow-up rate was $67 \%$ (68/ $102)$ and that for more than 1 year was $29 \%$ (30/102). The reasons for no treatment with follow-up were an obfuscated lesion in five patients, refusal of the patient in six cases, other primary advanced cancers in eight, and other serious disease in two patients. Among 34 patients with no treatment and no follow-up, the reasons were other primary advanced cancer in seven, poor nutrition in one, and unknown in 26 patients. Results of an endoscopic examination and progression are shown in $>$ Table2. Median follow-up periods for intramucosal and submucosal cancers were 26.5 and 16 months, respectively. Of 18 mucosal tumors, one showed DP and six showed LP. Of three submucosal tumors, two showed DP and three showed LP.

\section{Clinical course of two progressive cases}

\section{Patient 5}

The lesion was estimated to be submucosal cancer because of SMT (submucosal tumor)-like marginal elevation ( $\mathbf{F i g . 2 a ) . ~}$ Twelve months later, it was up to $100 \mathrm{~mm}$ and the depression progressed more deeply ( $\boldsymbol{\nabla}$ Fig. $\mathbf{2 b}$ ). Sixteen months later, the elevation became more obvious. The lesion was finally estimated to be cancer invading into the muscularis propria or deeper ( $\triangleright$ Fig. 2c).

\section{Patient 21}

The lesion was estimated to be submucosal cancer ( $>$ Fig. $3 a$ ) by the thickness and marginal elevation. Twelve months later, it was up to $50 \mathrm{~mm}$ and estimated to be muscularis propria cancer because the thickness and elevation became more obvious ( $\triangleright$ Fig.3b).

\section{Clinical course of a dormant case}

\section{Patient 11}

The lesion was initially diagnosed as intramucosal cancer, ( Fig.4a) and the diagnosis has remained mucosal cancer for 110 months ( $>$ Fig. 4 b).

\section{Discussion}

In our case series, only one of 18 mucosal tumors showed DP and six showed LP. Of three submucosal tumors, two showed DP and three showed LP.

Several studies have investigated the natural history of noninvasive gastric neoplasia based on histological follow-up data in a Western population. A report from Italy showed that $17 \%$ of noninvasive neoplasias had evolved to invasive GC during an average follow-up of 52 months [5]. A study from the Netherlands reported an annual incidence of invasive GC of $0.6 \%$ for mild-to-moderate dysplasia and $6 \%$ for severe dysplasia [6]. These data provide important information because intramucosal invasive cancer of the stomach, unlike intramucosal carcinoma of the colon, metastasizes. However, the distinction between intramucosal invasive cancer and HGN or carcinoma in situ can be challenging when evaluated by histological examination of the mucosal surface. Considering that these studies were based on biopsy specimens taken from the surface of mucosa, the reliability of differential diagnosis of invasive or noninvasive neoplasm is limited.

In another study, progression of EGC was investigated based on endoscopic follow-up data in a Japanese population [7]. This report from Osaka showed a cumulative 5 -year risk of progression to the advanced stage of $63.0 \%$ in 71 patients. However, in this study, initial depth of tumor invasion was not differentiated into mucosal and submucosal cancer. Because mucosal cancer in Japan includes noninvasive neoplasia, risk of progression to advanced cancer may differ substantially between mucosal and submucosal cancer. In the current study, only one of 18 mucosal cancers (mucosal HGN in Western countries) showed DP and six showed LP. These results suggest that a certain proportion of mucosal tumors can lie dormant for several years. 


\begin{tabular}{|c|c|c|c|c|c|c|c|c|c|c|c|c|}
\hline 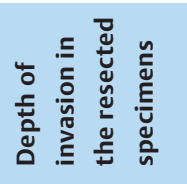 & $\Sigma$ & & & & & & & & & & & $\Sigma$ \\
\hline 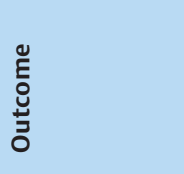 & 离 & $\begin{array}{l}\frac{5}{0} \\
\frac{5}{5} \\
\frac{5}{5}\end{array}$ & 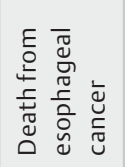 & 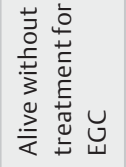 & $\begin{array}{l}\frac{5}{3} \\
0 \\
\frac{5}{5} \\
5\end{array}$ & 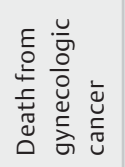 & 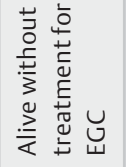 & 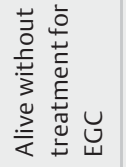 & 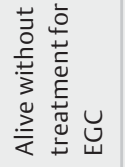 & 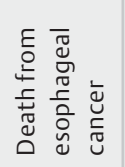 & 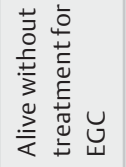 & 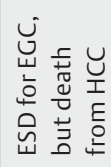 \\
\hline 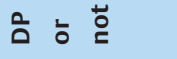 & $\stackrel{0}{z}$ & $\stackrel{0}{z}$ & io & $\stackrel{\circ}{z}$ & $\stackrel{\tilde{\nu}}{\nu}$ & $\stackrel{0}{z}$ & $\stackrel{0}{z}$ & $\stackrel{0}{z}$ & $\stackrel{0}{z}$ & $\stackrel{\tilde{x}}{x}$ & $\stackrel{0}{z}$ & $\stackrel{0}{z}$ \\
\hline এ ‘ & $\stackrel{\Xi}{\succ}$ & $\stackrel{0}{z}$ & $\stackrel{\varpi}{\succ}$ & $\stackrel{0}{z}$ & 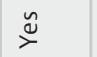 & $\stackrel{0}{z}$ & $\stackrel{0}{z}$ & 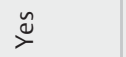 & $\stackrel{0}{z}$ & $\stackrel{y}{x}$ & $\stackrel{\circ}{z}$ & 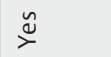 \\
\hline 苇 & $\stackrel{\bar{\rho}}{\risingdotseq}$ & $\stackrel{5}{\equiv}$ & $\stackrel{\pi}{\bar{n}}$ & $\stackrel{N}{\triangleq}$ & $\hat{\Xi}$ & $\stackrel{亠}{\circ}$ & $\supseteqq$ & $\supseteqq$ & $\supseteqq$ & $\stackrel{5}{\rho}$ & $\stackrel{2}{\rho}$ & 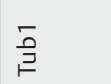 \\
\hline 華 & 0 & $\infty$ & $\stackrel{\llcorner}{\llcorner}$ & $\sigma$ & in & Ln & Ln & 0 & $\infty$ & $\stackrel{\circ}{\sim}$ & $\stackrel{\circ}{\sim}$ & 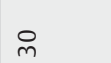 \\
\hline 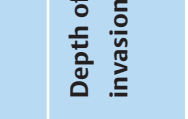 & $\Sigma$ & $\Sigma$ & $\Sigma$ & $\Sigma$ & in & $\Sigma$ & $\Sigma$ & $\Sigma$ & $\Sigma$ & $\Sigma$ & $\Sigma$ & $\sum_{n}^{2}$ \\
\hline 气̆ & $\stackrel{\mathbb{I}}{=}$ & $\stackrel{\mathbb{N}}{=}$ & $\stackrel{\varrho}{=}$ & 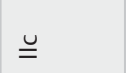 & $\stackrel{\varrho}{=}$ & 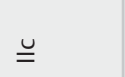 & 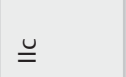 & $\stackrel{\varrho}{=}$ & $\stackrel{\varrho}{ }$ & $\stackrel{\mathbb{N}}{=}$ & $\stackrel{\mathbb{N}}{=}$ & $\stackrel{\varrho}{=}$ \\
\hline 节 & $\Sigma$ & $\supset$ & - & $\supset$ & $\Sigma$ & - & - & - & - & - & \lrcorner & - \\
\hline 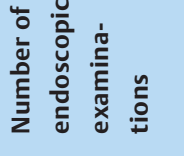 & $r$ & $\nabla$ & $\sim$ & $m$ & $\sim$ & $m$ & 6 & - & $m$ & $m$ & $\sigma$ & $m$ \\
\hline 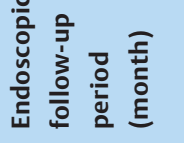 & $\tilde{m}$ & $\grave{\sim}$ & $\stackrel{m}{r}$ & $\stackrel{\stackrel{\sim}{N}}{ }$ & $\stackrel{\bullet}{\leftarrow}$ & 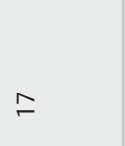 & $\stackrel{\infty}{m}$ & $\stackrel{\nabla}{\leftarrow}$ & $\stackrel{\Omega}{\neg}$ & $\stackrel{\bullet}{\leftarrow}$ & $\stackrel{\circ}{\rightleftharpoons}$ & $\stackrel{\bullet}{\leftarrow}$ \\
\hline 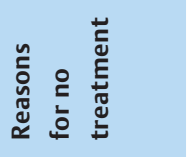 & 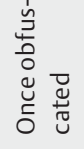 & 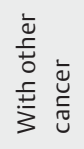 & 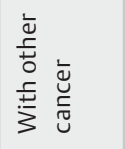 & 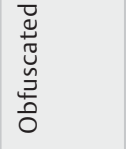 & 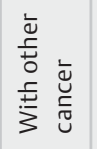 & 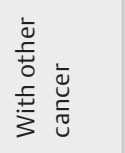 & 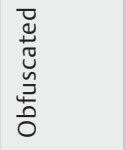 & 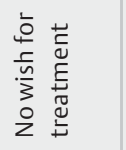 & 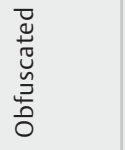 & 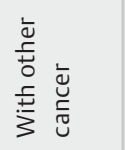 & 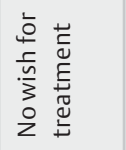 & 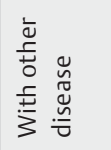 \\
\hline 夋 & g & n & $\pi$ & $\stackrel{\infty}{\infty}$ & $\stackrel{2}{\curvearrowright}$ & $\hat{0}$ & $\approx$ & $\stackrel{2}{\curvearrowright}$ & $\infty$ & $\approx$ & 6 & 品 \\
\hline ڤั̀ & $\Sigma$ & $\Sigma$ & $\Sigma$ & $\Sigma$ & $\Sigma$ & L & $\Sigma$ & $\Sigma$ & $\Sigma$ & $\Sigma$ & $\Sigma$ & $\Sigma$ \\
\hline נֶّ & - & $\sim$ & $m$ & $\nabla$ & in & 6 & $\wedge$ & $\infty$ & $\sigma$ & $\stackrel{\circ}{\sim}$ & $\mp$ & $\simeq$ \\
\hline
\end{tabular}



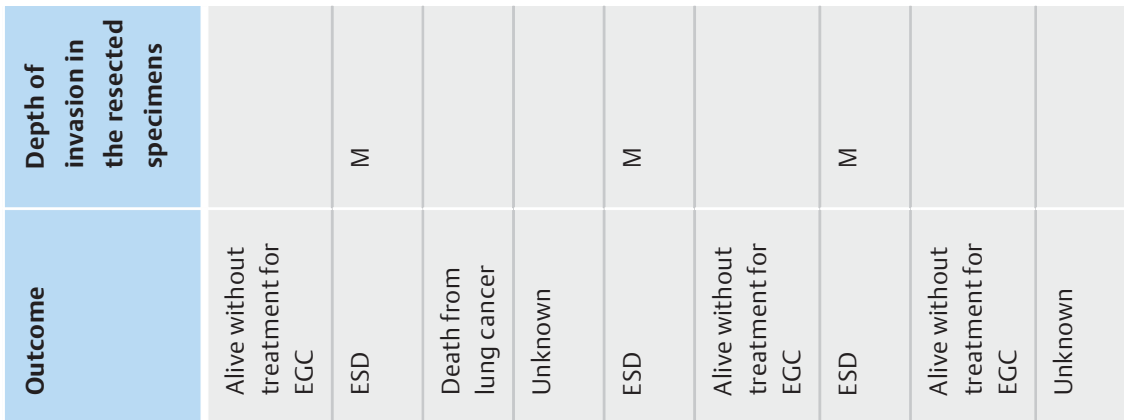

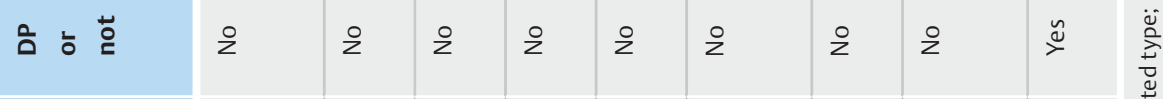

ヨ ‘

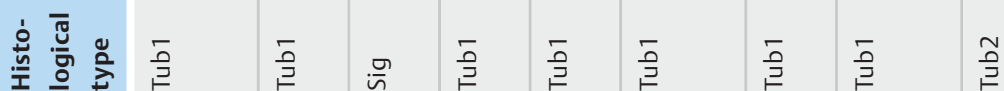

iั

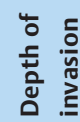

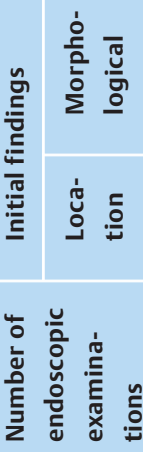

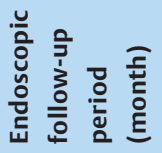

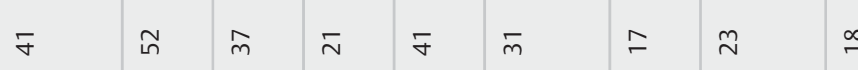

\begin{tabular}{|c|c|c|c|c|c|c|c|c|}
\hline 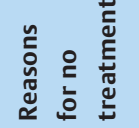 & 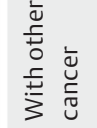 & 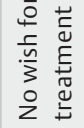 & 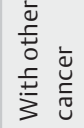 & 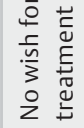 & 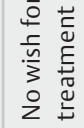 & 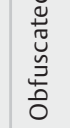 & 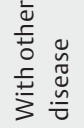 & 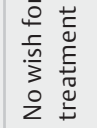 \\
\hline
\end{tabular}

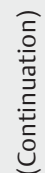

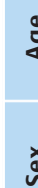

范

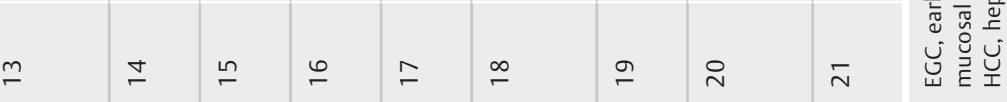




\begin{tabular}{|c|c|c|}
\hline & & $n=21$ \\
\hline \multicolumn{2}{|l|}{ Endoscopic follow-up period, median (range), months } & $23(13-110)$ \\
\hline \multicolumn{2}{|l|}{ Number of endoscopic examinations, median (range) } & $3(1-9)$ \\
\hline & $\begin{array}{l}\text { Mucosal cancers } \\
(n=18)\end{array}$ & $\begin{array}{l}\text { Submucosal cancers } \\
(n=3)\end{array}$ \\
\hline Lateral progression, $\mathrm{n}(\%)$ & $6(33)$ & $3(100)$ \\
\hline Deep progression, $\mathrm{n}(\%)$ & $1(6)$ & $2(67)$ \\
\hline Median degree of lateral progression & $50 \%$ & $67 \%$ \\
\hline Cumulative 1 - and 3-year risk for lateral progression & $6 \%$ and $31 \%$ & Both $100 \%$ \\
\hline Cumulative 1- and 3-year risk for deep progression & $0 \%$ and $6 \%$ & $33 \%$ and $100 \%$ \\
\hline
\end{tabular}
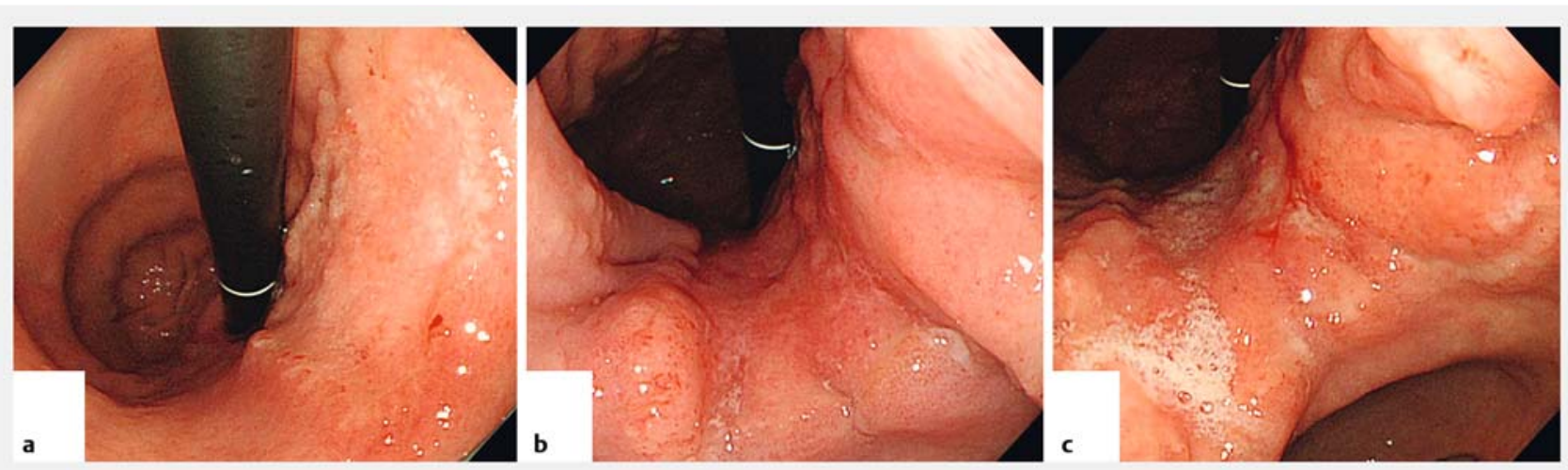

Fig. 2 Case 5: Lesion with lateral progression and deep progression (submucosal cancer to advanced cancer).

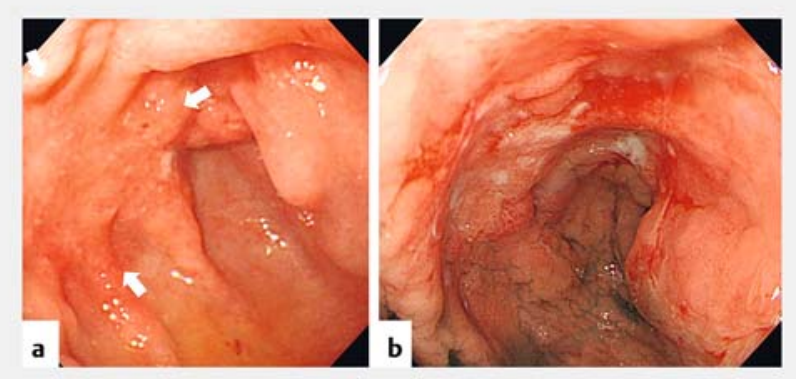

- Fig. 3 Case 21: Lesion with lateral progression and deep progression (submucosal cancer to advanced cancer).

When we consider the implications of DP and LP, DP is equivalent to advancement of the T category (i.e., T1a to T1b or T1b to T2). The T category is a main component of TNM staging and is associated with GC prognosis $[8,9]$. However, LP is not a component of TNM staging, and the relationship with prognosis remains unclear. However, lesion size is a significant predictive factor for metastasis [8] and may indirectly be associated with prognosis. Based on these findings, DP and LP are considered in oncological terms as worsening.
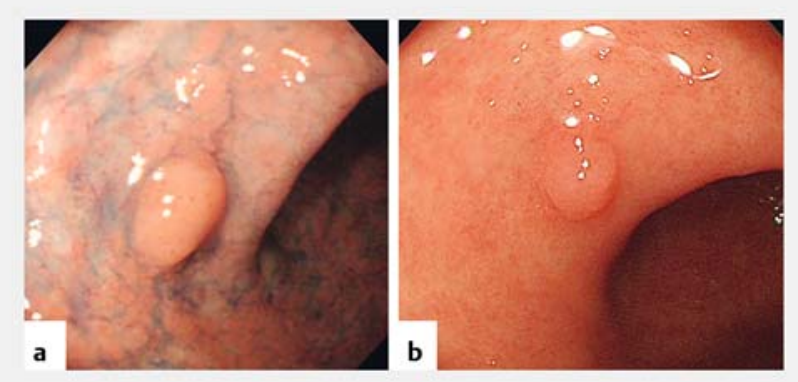

- Fig. 4 Case 11: The lesion was initially diagnosed as intramucosal cancer, and the diagnosis has remained mucosal cancer for 110 months.

The inclusion process for patients without treatment may be biased by some factors. A detailed database would help to identify all patients who fulfill the study criteria. Most hospitals may have a database of patients who have received treatment, but no such register for those not undergoing treatment. Another data source may then be used to identify the patients without treatment, such as medical charts or endoscopic records. However, untreated patients with short follow-up would be missed because they may have fewer follow-up visits or examinations. 
To overcome these shortcomings, we used the cancer registry to identify untreated patients with GC. This registry integrates histology, treatment, drug, and accounting databases, and includes dates of diagnosis and treatment. By combining multiple databases, the cancer registry can accumulate more patients than a single database and thus minimize the risk of overlooking patients with cancer. By referral to dates of diagnosis and treatment in the cancer registry, patients with no treatment or delayed treatment can be identified.

There are some limitations to the current study. The first limitation is the small number of patients. GC comprises a heterogeneous group of diseases with various histological types (differentiated or undifferentiated) and morphological types (elevated or depressed). Risk of progression may differ among these forms. However, the potential predictive factors for progression were not identified in our study because of the limited number of progressive cancers. A median follow-up of 23 months might be insufficient to clarify the natural history of GC, especially in revealing whether this type of cancer progresses to eventual death.

Another limitation is the low follow-up rate of $67 \%$ in this study. The follow-up rate, a standard index of completeness, is important for assessing the validity of a cohort study [10]. However, we consider the follow-up rate in this study was low because most patients with no treatment returned to previous doctors. The other limitation is that the judgement of DP, particularly for progression from submucosal cancer to proper muscle invasion, is difficult and can be subjective.

\section{Conclusion}

In conclusion, our study suggests that a certain proportion of mucosal cancers can lie dormant for several years. Further large-scale studies in a multicenter setting should help to overcome the limitations of this study.
Competing interests

None

References

[1] Fujisaki J, Nakajima T, Hirasawa T et al. Natural history of gastric cancer-a case followed up for eight years: early to advanced gastric cancer. Clin J Gastroenterol 2012; 5: 351 - 354

[2] Iwai T, Yoshida M, Ono $\mathrm{H}$ et al. Natural history of early gastric cancer: a case report and literature review. J Gastric Cancer 2017; 17: 88 - 92

[3] Abe S, Oda I, Shimazu T et al. Depth-predicting score for differentiated early gastric cancer. Gastric Cancer 2011; 14: 35-40

[4] Tsujii Y, Kato M, Inoue T et al. Integrated diagnostic strategy for the invasion depth of early gastric cancer by conventional endoscopy and EUS. Gastrointest Endosc 2015; 82: 452 - 459

[5] Rugge M, Cassaro M, Di Mario F et al. The long term outcome of gastric non-invasive neoplasia. Gut 2003; 52: $1111-1116$

[6] de Vries AC, van Grieken NC, Looman CW et al. Gastric cancer risk in patients with premalignant gastric lesions: a nationwide cohort study in the Netherlands. Gastroenterology 2008; 134: 945 - 952

[7] Tsukuma H, Oshima A, Narahara $\mathrm{H}$ et al. Natural history of early gastric cancer: a non-concurrent long term follow up study. Gut 2000; 47: $618-621$

[8] Cambruzzi E, Azeredo AM, Kronhart A et al. The presence of metastases in regional lymph nodes is associated with tumor size and depth of invasion in sporadic gastric adenocarcinoma. Arq Bras Cir Dig 2014; 27: $18-21$

[9] Im W], Kim MG, Ha TK et al. Tumor size as a prognostic factor in gastric cancer patient. J Gastric Cancer 2012; 12: 164 - 172

[10] Xue X, Agalliu I, Kim MY et al. New methods for estimating follow-up rates in cohort studies. BMC Med Res Methodol 2017; 155: 1-10 